\title{
MEASUREMENT SYSTEM FOR FRACTIONAL GLOW TECHNIQUE
}

\author{
A. Pietkun, L. Polakiewicz and H.L. Oczkowski \\ Institute of Physics, N. Copernicus University, Grudziądzka 5/7, 87-100 Toruń, Poland \\ (Received March 10, 1992; in final form May 4, 1992)

\begin{abstract}
Some details of computerized arrangement for recording fractional thermoluminescence at high temperatures are presented. The investigation of energy distribution of traps for quartz crystals exemplifies the measurement procedure and data handling.
\end{abstract}

PACS numbers: $78.60 . \mathrm{Kn}$

\section{Introduction}

Fractional glow technique initiated by Gobrecht and Hofmann [1] seems to be the most obvious method of trap distribution spectrum estimation [2]. However, there are not many laboratories which use this method as a common way of trap depth problem treatment. The reason are rather high requirements of measurement procedure, its time consumption and huge number of experimental data needed for satisfactory results of thermal analysis [3-5].

As the knowledge of thermoluminescence phenomenon mechanism in a particular mineral can be the only basis of reasonable work in the thermoluminescence dating or dosimetry field, it is suggested to exploit the fractional glow technique (FGT) more intensively. For such a goal a new computer-controlled system for thermoluminescence investigation of minerals in the high region of temperature was built. Some introductory experimental results are presented.

Details of experimental arrangement for recording fractional thermoluminescence excited at liquid nitrogen temperature were previously described in our paper [6]. The data handling and error estimation procedure used in our laboratory were therein also presented in detail, so the reader is referred to this paper [6].

\section{Experimental device}

The high-temperature thermoluminescence unit is a steel cylindrical cell with U-shaped heating strip (kanthal). The cell is water-cooled and allows to investigate thermoluminescence in the range from room temperature (RT) to $700 \mathrm{~K}$. 
The heating proceeds in the high purity nitrogen atmosphere. The temperature control is realized by means of an IBM/XT computer on-line which is equipped with PCLab-718 High Performance Acquisition Card. The computer analog signal transformed by $\mathrm{U} / \mathrm{f}$ converter into rectangular pulses controls a heating supply. The linear heating rate from 0.1 to $10 \mathrm{~K} / \mathrm{s}$ is achieved by means of closed-loop PID control or is generated by previously found heating algorithm. The temperature is measured using the $\mathrm{NiCr}-\mathrm{Ni}$ thermocouple. Figure 1 presents the thermoluminescence glow curve of quartz obtained for linear heating with the rate of $1 \mathrm{~K} / \mathrm{s}$.

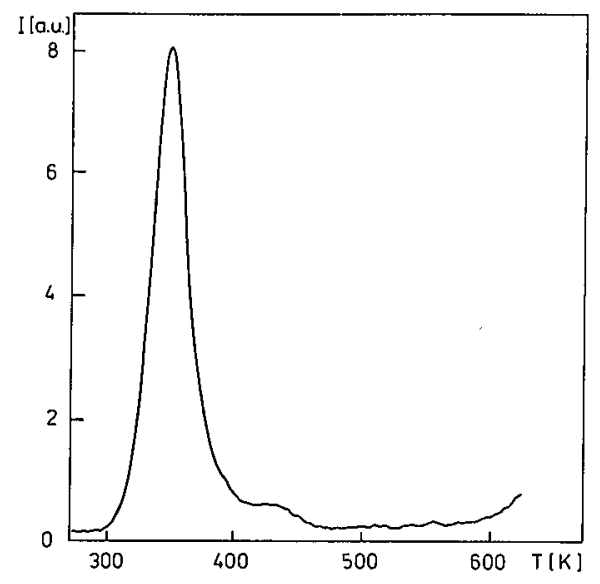

Fig. 1. Glow curve of quartz crystal excited by X-ray irradiation.

The light detection circuit consists of a photomultiplier (EMI 6097B), a chopper and a lock-in amplifier (UNIPAN-232B). A water filter and a blue optical filter (BG-12) protect the phototube from the thermal emission. The chopper unit installed in front of the phototube provides a reference signal. The lock-in nanovoltmeter selects the low-noise signal from photomultiplier output and sends it to the computer input. Figure 2 shows a blok diagram of the system.

The FGT is realized by the series of successive linear heating and cooling cycles. The temperature oscillations have fixed increasing and decreasing temperature amplitudes and continuously enhanced average temperature of heating. For small temperature oscillation of $i$-th cycle the thermoluminescence intensity $I_{i}$ is exponentially dependent on temperature [1]:

$$
I_{i}=A_{i} \exp \left(-E_{i} / k T\right)
$$

where $T-$ temperature, $A_{i}-$ constant and $k-$ Boltzmann constant. $E_{i}$ corresponds to the averaged energy of the fraction of traps emptied during $i$-th cycle. The energy resolution power of FGT depends on the difference between heating and cooling amplitude.

In the presented equipment the measurement procedure is generated by a program written in Quick Basic. It allows to regulate heating process and measure the thermoluminescence intensity five times per second. The fixed temperature 


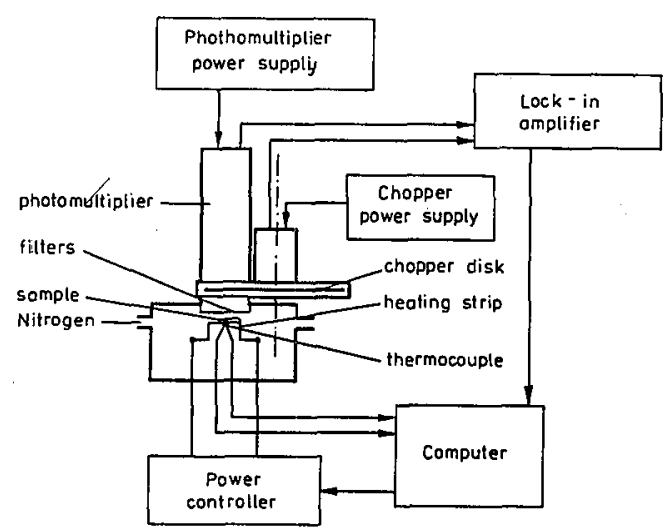

Fig. 2. Block diagram of experimental device for the fractional glow technique.

program instead of closed-loop control is preferred, because there is no danger of disturbances caused by bad thermocouple-strip thermal contact.

After each cycle the acquired data, the temperature and thermoluminescence intensity, are written on a disk. Figure 3 represents the fractional glow curve which can be observed on the computer display during the experiment. Samples of natural quartz crystals cut into $0.5 \mathrm{~mm}$ slabs were used for all testing measurements.

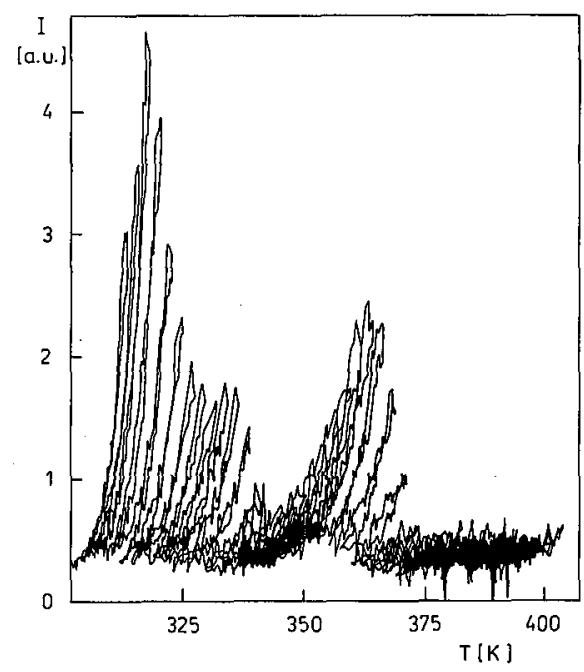

Fig. 3. An example of the fractional glow curve for temperature range RT-560 K consisting of 35 cycles, the temperature step $-5 \mathrm{~K}$. Two quartz peaks are clear $(300 \mathrm{~K}$, $380 \mathrm{~K})$. The measurement is performed immediately after excitation. 


\section{Data handling}

The first step of data handling is smoothing of the light intensity dependence. The comparison of heating and cooling curves shapes in order to detect measurement irregularities [1] is also performed. The formula

$$
\ln I_{i}=\text { const }-\left(E_{i} / k T\right)
$$

is the basis of linear regression for energy and standard error estimation for heating and cooling part of each cycle. The energies $\left(E_{i}\right)$ and corresponding partial light sums $\left(L_{i}\right)$ are the data for the density spectrum of populated traps calculation. Figure 4 shows an example of trap energy dependence on the cycle number after repeated triple smoothing procedure.

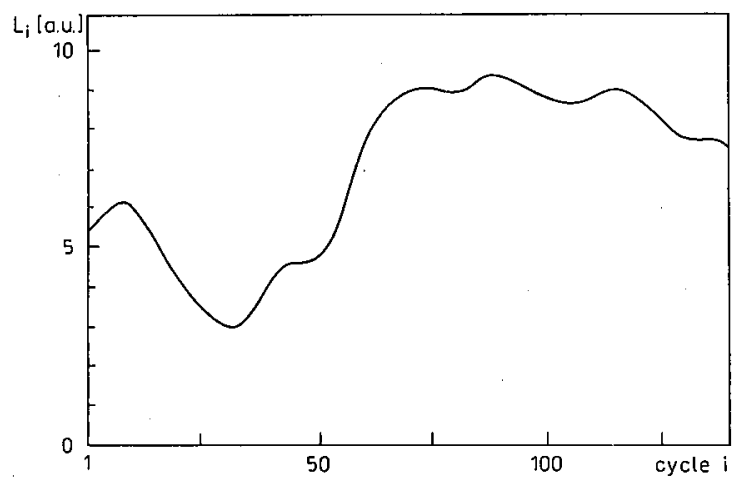

Fig. 4. The smoothed trap energy dependence on the cycle number obtained from the fractional glow curve in the range 300 to $570 \mathrm{~K}$ for quartz; 140 cycles; temperature step $2 \mathrm{~K}$.

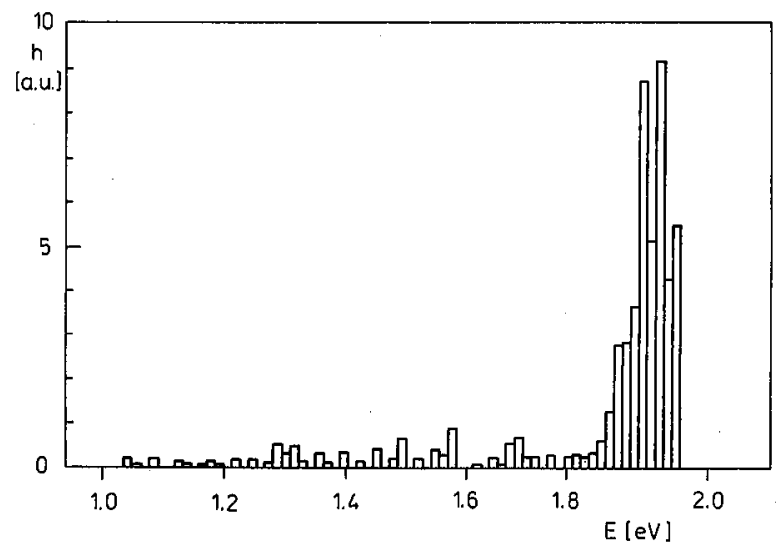

Fig. 5. The density spectrum of populated traps. The result for the same FGT measurement which is presented in Fig. 4. 
The ratio

$$
h_{i}=L_{i} /\left|E_{i+1}-E_{i}\right|
$$

represents the share of trap emptying during $i$-th cycle in the density spectrum. All details of calculations leading to the density spectrum of populated traps are described in [6].

Figure 5 examplifies a histogram obtained for a natural quartz crystal. The histogram was calculated with the step of $20 \mathrm{meV}$ from the only one fractional curve.

\section{Final remarks}

The presented computer on-line system gives possibility of efficient estimation of density spectrum of populated traps in crystals. The flexibility of arrangement and data operating program enables to obtain quantitative results after a series of experiments with different heating rates [2]. The other methods of trap parameters of thermoluminescence kinetics investigations can be applied and developed.

\section{Acknowledgments}

In the course of technical works J. Kowalski, H. Schiller and S. Maciejewski gave us a substantial help in construction of many details of the presented apparatus. The support for this work was provided by the Ministry of National Education (grant RPBP I.11 A.1.5.1) and our University (grant 613F).

\section{References}

[1] H. Gobrecht, D. Hofmann, J. Phys. Chem. Solids 27, 509 (1966).

[2] S.W.S. McKeever, Thermoluminescence of Solids, Cambridge University Press, Cambridge 1985.

[3] H.L. Oczkowski, J. Lumin. 17, 113 (1978).

[4] H.L. Oczkowski, Phys. Status Solidi A 68, 199 (1981).

[5] F. Firszt, H.L. Oczkowski, Phys. Status Solidi $A$ 111, K113 (1989).

[6] H.L. Oczkowski, Acta Phys. Pol. A76, 649 (1989). 